\title{
"Fetomaternal Outcome in Term Oligohydramnios-A Study in Rajshahi Medical College Hospital, Rajshahi, Bangladesh"
}

\author{
Dr. Homaira Shahreen (Simi) ${ }^{*}$ \\ Assistant Professor (Obst \& Gynae), Rajshahi Medical College Hospital, Rajshahi, Bangladesh
}

DOI: $10.36348 /$ sijog.2020.v03i11.002

| Received: 10.11.2020 | Accepted: 19.11.2020 | Published: 25.11 .2020

*Corresponding author: Dr. Homaira Shahreen

\section{Abstract}

Introduction: Oligohydramnios is defined as AFI $<5 \mathrm{~cm}$ (amniotic fluid index) or the maximum vertical pocket of liquor is less than $2 \mathrm{~cm}$. It has been correlated with increased risk of intrauterine growth retardation, meconium aspiration, severe birth asphyxia, low APGAR score and congenital abnormalties. There is an inverse relationship between amniotic fluid volume and pregnancy outcome. Objective: To find out the fetomaternal Outcome in Term Oligohydramnios. Materials and Methods: This study was conducted in the department of Obstetrics and Gynaecology at Rajshahi Medical College Hospital, Rajshahi, Bangladesh over a period of 1 year from January 2018 to December 2018. 65 patients in third trimester of pregnancy with oligohydramnios were selected randomly after satisfying inclusion and exclusion criteria. Results: This study was performed in 65 pregnant women with amniotic fluid index $<5 \mathrm{~cm}$ with sinleton pregnancy. In this study 38 out of the 65 patients (58\%) had vaginal delivery (either induced or spontaneous) and 27 patients (42\%) underwent lower segment caesarean section. Patients with amniotic fluid index $4-5 \mathrm{~cm}$ on USG were $39(60 \%)$ followed by AFI $2-4 \mathrm{~cm}$ was $18(28 \%)$ and AFI $<2 \mathrm{~cm}$ was $8(12 \%)$. Low birth weight $(<2.5 \mathrm{~kg})$ was seen in 19 newborns $(29 \%)$. NICU was required in 22 newborns (34\%). Fetal congenital anomalies were seen in 4 cases (4\%). congenital anomalies were hydronephrosis, diaphragmatic hernia, hypoplastic kidneys and multiple congenital anomalies. Conclusion: In conclusion, oligohydramnios in term pregnancies is associated with an increased risk of obstetric intervention. All cases of oligohydramnios require intensive antepartum and intrapartum care. Early detection of oligohydramnios, evaluation of the cause and severity of oligohydramnios and assessment of gestational age are essential for management. Its management may help in reduction of perinatal morbidity and mortality and decreased caesarean deliveries.

Keywords: Oligohydramnios, Liquor, Intrauterine, Pregnant, Women.

Copyright $\odot$ 2020 The Author(s): This is an open-access article distributed under the terms of the Creative Commons Attribution 4.0 International License (CC BY-NC 4.0) which permits unrestricted use, distribution, and reproduction in any medium for non-commercial use provided the original author and source are credited.

\section{INTRODUCTION}

The amniotic fluid baths the fetus is necessary for its proper growth and development. Amniotic fluid is a clear, slightly yellowish liquid that surrounds the fetus in the amniotic sac during pregnancy. Amniotic fluid is produced after the amniotic sac is formed at about 12 days after conception. It is first made up of effusion by the mother's circulation and then around the 20 weeks fetal urine becomes the primary substance [1].The fluid is faintly alkaline with low specific gravity of 1.010. Volume of amniotic fluid decreases with increasing gestational age. It measures about $50 \mathrm{ml}$ at 12 weeks, $400 \mathrm{ml}$ at 20 weeks and reaches peak of 1 liter at 36-38 weeks thereafter the amount diminishes and at term it measures about $600-800 \mathrm{ml}$ [2]. It cushions the fetus from physical trauma, permits fetal lung growth, and provides a barrier against infection during pregnancy; it acts as a shock absorber, helps in growth and development by regulating temperature, providing nutrition, avoiding external injuries and impact of uterine contractions and prevents adhesion between fetal parts and amniotic sac. During labour, the amnion and chorion are combined to form a hydrostatic wedge which helps in dilatation of cervix; it prevents and guards against umbilical cord compression. Amniotic fluid volume is assessed by amniotic fluid index (AFI) using four duadrant technique during transabdominal USG [3]. Decrease in amniotic fluid volume is known as oligohydramnios. Oligohydramnios is defined as AFI $<5 \mathrm{~cm}$ (amniotic fluid index) or the maximum vertical pocket of liquor is less than $2 \mathrm{~cm}$. 
The common clinical features are smaller symphysiofundal height, fetal malpresentation, and undue prominence of fetal parts and reduced amount of amniotic fluid. The incidence of oligohydramnios is $0.5-1 \%$ of all pregnancies [4]. It has been correlated with increased risk of intrauterine growth retardation, meconium aspiration, severe birth asphyxia, low APGAR score and congenital abnormalities. It can lead to deformation syndromes such as cranial, facial or skeletal abnormalities, pulmonary hypoplasia, urinary tract malformations and adverse perinatal outcome [5]. Oligohydramnios isassociated with maternal morbidity in terms of increased rates of induction and operative interventions. Oligohydramnios is also associated with a variety of ominous pregnancy outcomes such as prenatal death, fetal distress in labor, poor infant condition and structural fetal anomalies. The lower the amniotic fluid volume, the greater the incidence of perinatal morbidity and mortality. There is an inverse relationship between amniotic fluid outcome and pregnancy outcome [6].

\section{MATERIALS AND METHODS}

This study was conducted in the department of Obstetrics and Gynaecology at Rajshahi Medical College Hospital, Rajshahi, Bangladesh over a period of 1 year from January 2018 to December 2018.65 patients in third trimester of pregnancy with oligohydramnios were selected randomly after satisfying inclusion and exclusion criteria.

\section{Inclusion Criteria}

a) Antenatal patients in their third trimester with intact membranes, amniotic fluid index $<5$, singleton pregnancy

\section{Exclusion Criteria}

a) Antenatal patients having heart diseases, polyhydramnios, premature rupture of membranes, twins and multiple pregnancies, diabetic patient, maternal hypertensive disease.
A detailed history and examination were done. All required investigation done. Amount of liquor amnii in the present study was assessed by ultrasonography. Though there are various methods for sonographic assessment of amniotic fluid, amniotic fluid index (AFI) was taken as the criteria. Routine management in form of rest, left lateral position, oral and intravenous hydration and control of etiological factor was done if present. Fetal surveillance was done by USG, modified Biophysical profile and Doppler. Decision of delivery by either induction or elective or emergency LSCS was done as per required. Some patients were already in labour and other allows going in spontaneous labour. Cases were than studied for maternal and perinatal outcome.

\section{OBSERVATIONS AND RESULTS}

This study was performed in 65 pregnant women with amniotic fluid index $<5 \mathrm{~cm}$ with sinleton pregnancy. Patients with amniotic fluid index $4-5 \mathrm{~cm}$ on USG was $39(60 \%)$ followed by AFI 2-4 cm was 18 $(28 \%)$ and AFI $<2 \mathrm{~cm}$ was $8(12 \%)$ [Table 1]. Colour of amniotic fluid Clear 47 (72.0\%), Thin Meconium 15 (23.0\%) and Thick Meconium 3 (5.0\%) [Table-2]. In this study 38 out of the 65 patients $(58 \%)$ had vaginal delivery (either induced or spontaneous) and 27 patients (42\%) underwent lower segment caesarean section [Table 3]. The most common indication for cesarean section was severe oligohydramnios $(30 \%)$ followed by fetal distress (22\%), failure of induction (18.5\%), severe IUGR (18.5\%) and malpresentations (11\%). Meconium stain liquor was seen in 18 patients $(28 \%)$ out of 65 patients [Table 4]. Incidence of low birth weight babies $(<2.5 \mathrm{~kg})$ was seen in 19 newborns (29\%). APGAR (appearance, pulse, grimace, activity, respiration) score was calculated in all newborns at 5 minutes. 17 babies had APGAR score $<7$ at 5 minutes. NICU was required in 22 newborns (34\%) [Table 5]. Fetal congenital anomalies were seen in 4 cases $(6 \%)$. congenital anomalies were hydronephrosis (1.5\%), diaphragmetic hernia $(1.5 \%)$, hypoplastic kidneys $(1.5 \%)$ and multiple congenital anomalies (1.5\%) [Table 6].

Table-1: Amniotic Fluid Index ( $=65$ )

\begin{tabular}{|l|r|r|r|}
\hline S. No. & AFI in cm & $\begin{array}{r}\mathbf{N} \\
\text { umber }\end{array}$ & Percentage \\
\hline 1 & $<2$ & 8 & 12.0 \\
\hline 2 & $2-4$ & 18 & 28.0 \\
\hline 3 & $4-5$ & 39 & 60.0 \\
\hline Total & & 65 & 100.0 \\
\hline
\end{tabular}




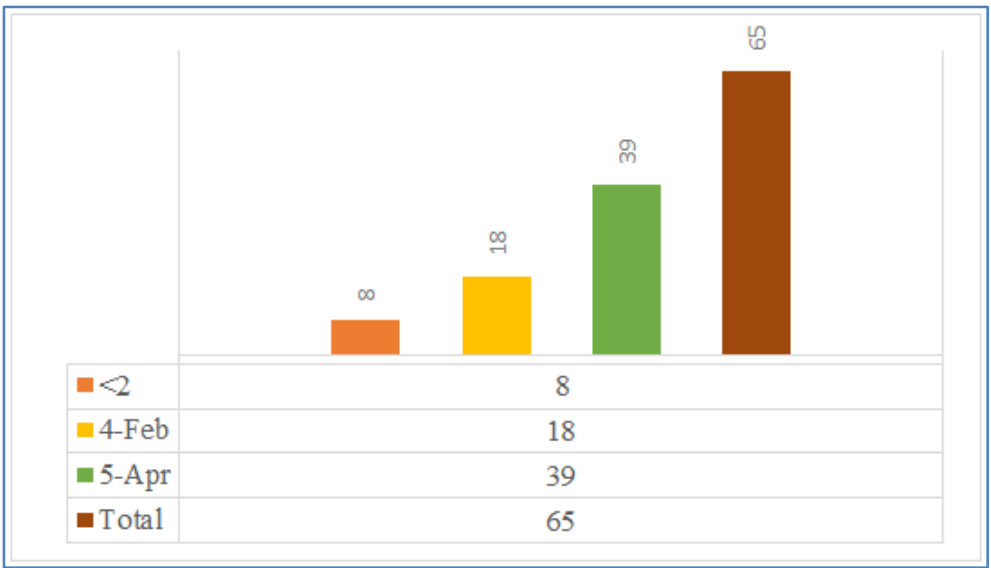

Fig-1: Amniotic Fluid Index

Table-2: Colour of amniotic fluid ( $N=65)$

\begin{tabular}{|l|l|l|l|}
\hline S. No. & Liquor & Number & Percentage \\
\hline 1 & Clear & 47 & 72.0 \\
\hline 2 & Thin Meconium & 15 & 23.0 \\
\hline 3 & Thick Meconium & 3 & 5.0 \\
\hline Total & & 65 & 100.0 \\
\hline
\end{tabular}

Table-3: Mode of Delivery ( $N=65$ )

\begin{tabular}{|l|c|l|l|l|}
\hline S.No. & Mode of delivery & & Number & Percentage \\
\hline \multirow{2}{*}{1} & \multirow{2}{*}{ Vaginal } & Spontaneous & 12 & 18.0 \\
\cline { 3 - 5 } & & Induced & 26 & 40.0 \\
\hline 2 & Cesarean Section & & 27 & 42.0 \\
\hline Total & & & 65 & 100.0 \\
\hline
\end{tabular}

Table-4: Indications of LSCS ( $N=27)$

\begin{tabular}{|l|l|l|l|}
\hline S. No. & Indications & Number & Percentage \\
\hline 1 & Severe Oligohydramnios & 8 & 30.0 \\
\hline 2 & Fetal Distress & 6 & 22.0 \\
\hline 3 & Failure of induction & 5 & 18.5 \\
\hline 4 & Serere IUGR & 5 & 18.5 \\
\hline 5 & Malpresentation & 3 & 11.0 \\
\hline Total & & 27 & 100.0 \\
\hline
\end{tabular}

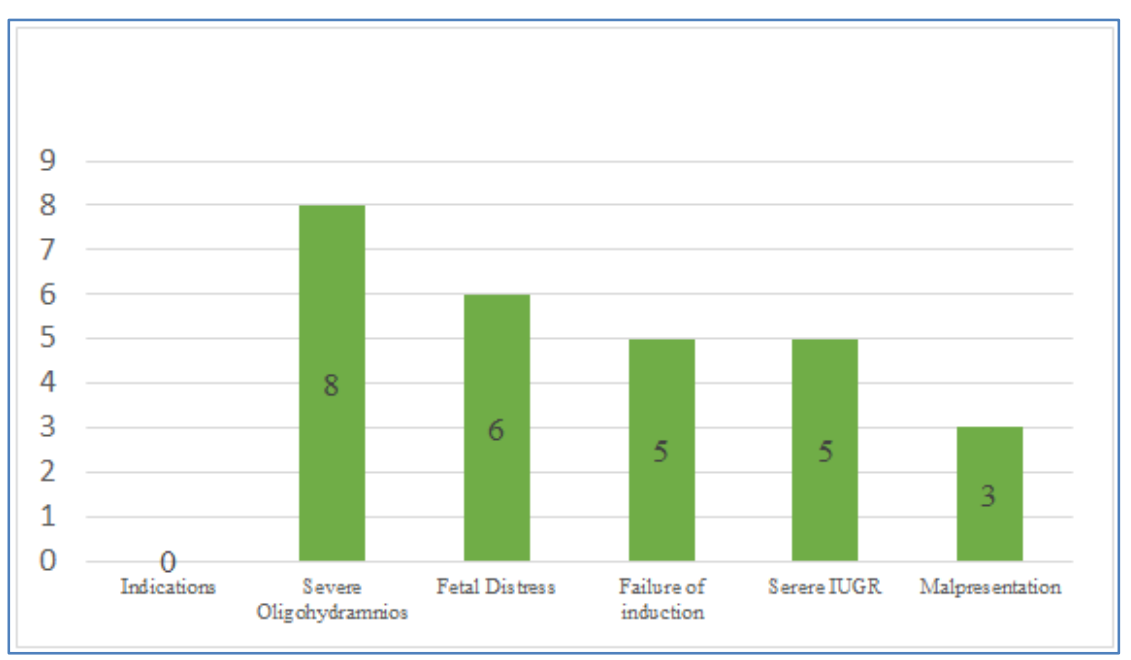

Fig-2: Indications of LSCS 
Table-5: Neonatal outcome $(\mathrm{N}=22)$

\begin{tabular}{|l|l|l|l|}
\hline S. no. & Neonatal Outcome & Number & Percentage \\
\hline 1 & Birth weight $<2.5 \mathrm{Kg}$ & 19 & 29.0 \\
\hline 2 & APGAR $<7$ at 5 minutes & 17 & 26.0 \\
\hline 3 & NICU admission & 22 & 34.0 \\
\hline
\end{tabular}

Table-6: Congenital Malformations ( $=4$ )

\begin{tabular}{|l|l|l|l|}
\hline S. No. & Congenital Malformations & Number & Percentage \\
\hline 1 & Hydronephrosis & 1 & 1.5 \\
\hline 2 & Diaphragmatic Hernia & 1 & 1.5 \\
\hline 3 & Hypoplastic kidneys & 1 & 1.5 \\
\hline 4 & Multiple congenital anomalies & 1 & 1.5 \\
\hline Total & & 4 & 6.0 \\
\hline
\end{tabular}

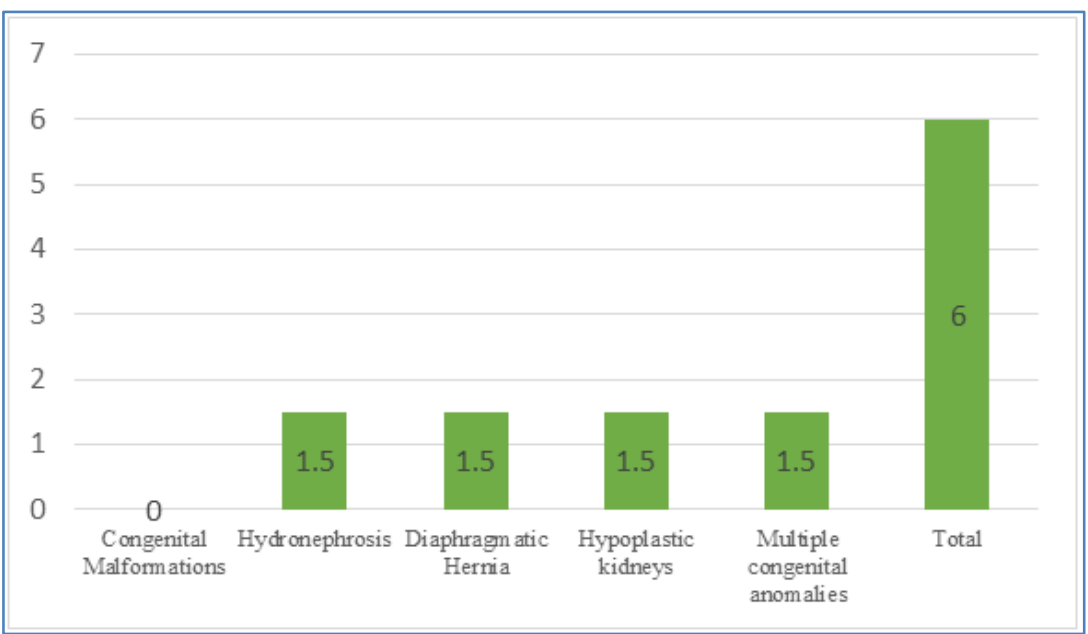

Fig-3: Congenital Malformations

\section{DISCUSSION}

The primary objective of the antenatal screening is to detect any condition which can lead to a high risk pregnancy. Ultrasound examination during that period is a sensitive and reliable method of assessing the amniotic fluid and to detect oligohydramnios or polyhydramnios. In the present study, 38 out of the 65 patients $(58 \%)$ had vaginal delivery (either induced or spontaneous) and 27 patients $(42 \%)$ underwent lower segment caesarean section. The most common indication was severe oligohydramnios (30\%) followed by fetal distress (22\%), failure of induction (18.5\%), severe IUGR $(18.5 \%)$ and malpresentations (11\%).Similar study conducted at Sir Gangaram Hospital [7] shows 68\% vaginal deliveries in induced patients of oligohydramnios and $32 \%$ by caesarean section which is comparable to our study. Manzanares S et al. [8] conducted a study which shows $84 \%$ vaginal deliveries in patients induced for oligohydramnios and $16 \%$ caesarean sections. Similar findings were seen in study conducted by Bangal V B et al. [9] that $56 \%$ of oligohydramnios women had spontaneous vaginal delivery and $44 \%$ had operative/assisted delivery. Sowmya $\mathrm{K}$ et al. [10] observed that in patients with oligohydramnios, $50 \%$ underwent caesarean section for fetal distress, $26.47 \%$ for intrauterine growth restriction (IUGR) with doppler changes, $14.7 \%$ for failed induction and $8.82 \%$ for cephalopelvic disproportion. Incidence of low birth weight baby $<2.5 \mathrm{~kg}$ was seen in 19 newborns $(29 \%)$. APGAR (appearance, pulse, grimace, activity, respiration) score was calculated in all newborns at 5 minutes. 17 babies had APGAR score $<7$ at 5 minutes. NICU was required in 22 newborns (34\%). Fetal congenital anomalies were seen in 4 cases $(6 \%)$. congenital anomalies were hydronephrosis (1.5\%), diaphragmatic hernia (1.5\%), hypoplastic kidneys $(1.5 \%)$ and multiple congenital anomalies (1.5\%). In study by Sowmya K et al. [10] low birth weight was seen in $48 \%$, Apgar score <7 seen in $14 \%$ and $14 \%$ were admitted in NICU. Another study by Madhavi K et al. [11] found the incidence of meconium stained liquor in 36\%, 20\% had Apgar score less than 7 at 5 minutes, incidence of NICU admission in $34 \%$, incidence of meconium aspiration syndrome (MAS) in $6 \%$. In a study conducted by Guin G et al. [12] the congenital malformations were bilateral polycystic kidneys, hydronephrosis with hydroureter and omphalocele. In our study the most common congenital anomaly was hydronephrosis.

\section{CONCLUSION}

In conclusion, oligohydramnios in term pregnancies is associated with an increased risk of obstetric intervention. All cases of oligohydramnios require intensive antepartum and intrapartum care. 
Early detection of oligohydramnios, evaluation of the cause and severity of oligohydramnios and assessment of gestational age are essential for management. Its management may help in reduction of perinatal morbidity and mortality and decreased caesarean deliveries. In the presence of oligohydramnios, the occurrence of non-reactive NST, thick meconium stained liquor, development of fetal distress, the rate of LSCS, low Apgar score, low birth weight, perinatal Bangladesh. 2011; 61(6): 652-655. Morbidity and mortality are more. Determination of AFI is a valuable screening test for predicting fetal distress in labor. Due to intrapartum complications and high rate of perinatal morbidity and mortality, rates of caesarean section are rising, but decision between vaginal delivery and caesarean section should be well balanced so that unnecessary maternal morbidity is prevented and other side timely intervention can reduce perinatal morbidity and mortality.

\section{REFERENCES}

1. Gary Cunningham, F., Kenneth, J. Leveno, Steven, L. (2014). Bloom. Williams's obstetrics.24th edn.2014.

2. Dutta, D. C. (2015). Textbook of Obstetrics, 8th edition, 43-44; 250-251.

3. Phelan, J. P., Smith, C. V., \& Brov Ssaid, P. (1987). Amniotic fluid volume assesment using the four quadrant technique in the pregnancy between 36 and 42 week. J Reprod med, 32, 540.

4. Casey, B. M., McIntire, D. D., Bloom, S. L., Lucas, M. J., Santos, R., Twickler, D. M., ... \& Leveno, K. J. (2000). Pregnancy outcomes after antepartum diagnosis of oligohydramnios at or beyond 34 weeks' gestation. American journal of obstetrics and gynecology, 182(4), 909-912.
5. King, J. C., Mitzner, W., Butterfield, A. B., \& Queenan, J. T. (1986). Effect of induced oligohydramnios on fetal lung development. American journal of obstetrics and gynecology, 154(4), 823-828.

6. Rutherford, S. E., Phelan, J. P., Smith, C. V., \& Jacobs, N. A. N. C. Y. (1987). The four-quadrant assessment of amniotic fluid volume: an adjunct to antepartum fetal heart rate testing. Obstetrics and gynecology, 70(3 Pt 1), 353-356.

7. Umber, A. (2009). Perinatal Outcome in Pregnancies Complicated by Isolated Oligohydramnios at. Annals of King Edward Medical University, 15(1), 35-35.

8. Manzanares, S., Carrillo, M. P., González-Perán, E., Puertas, A., Montoya, F., Manzanares, S., ... \& Montoya, F. (2007). Isolated oligohydramnios in term pregnancy as an indication for induction of labor. The Journal of Maternal-Fetal \& Neonatal Medicine, 20(3), 221-224.

9. Bangal, V. B., Giri, P. A., \& Sali, B. M. (2011). Incidence of oligohydramnios during pregnancy and its effects on maternal and perinatal outcome. $J$ Pharm Biomed Sci (JPBMS), 12(05), 1-4.

10. Sowmya, K., Varghese, B., \& Umashankar, B. Y. (2014). Effect of isolated oligohydramnios in otherwise normal term pregnancy. International Journal of Biomedical Research ISSN: Journal DOI, 10.

11. Madhavi, K., \& Rao, P. C. (2015). Clinical study of oligohydramnios, mode of delivery and perinatal outcome. IOSR Journal of Dental and Medical Sciences, 14(4), 6-11.

12. Guin, G., Punekar, S., Lele, A., \& Khare, S. (2011). A prospective clinical study of fetomaternal outcome in pregnancies with abnormal liquor volume. The journal of Obstetrics and gynecology of India, 61(6), 652-655. 